\title{
2.5 INDIVIDUAL RADIO PULSES FROM NP 0531
}

\author{
J. M. SUTTON \\ NRAO, Green Bank, W. Va., U.S.A.* and IOTA, University of Cambridge, U.K. \\ and \\ D. H.STAELIN and R. M. PRICE \\ Massachusetts Institute of Technology, Cambridge, Mass., U.S.A.
}

\begin{abstract}
Observations with a swept frequency machine show that the shapes of individual pulses from NP 0531 are truncated exponentials with widths proportional to $\lambda \sim 4$, consistent with the broadening arising from scattering in the interstellar medium. A histogram of pulse intensity reveals two different populations. The very strong pulses are associated only with the main pulse and interpulse.
\end{abstract}

We present further observations of individual pulses from the Crab Nebula pulsar (NP 0531) obtained using a swept frequency machine on the $300 \mathrm{ft}$ radiotelescope at Green Bank. The technique has been described previously by Staelin and Sutton (1970) and Sutton et al. (1970). Briefly, to achieve a given time resolution it is necessary to use a bandwidth narrower than a critical value determined by the dispersion measure and frequency of observation. The combination of bandwidth and time resolution restricts the sensitivity such that observations of individual pulses are limited to strong pulses from particular pulsars. The sensitivity can be considerably improved by tracking the pulses as they sweep in frequency using individual channels of a filter bank to track different parts of the pulse. For all our observations the polarization is circular.

Radiation from NP 0531 at metre wavelengths is characterized by the occasional very strong pulses which first led to its discovery (Staelin and Reifenstein, 1968). We have already reported observations of individual strong pulses at $115 \mathrm{MHz}$ and $157 \mathrm{MHz}$ (Staelin and Sutton, 1970) obtained by triggering the swept frequency machine on strong pulses previously detected at a slightly higher frequency. Figure 1 shows some further pulse shapes of higher resolution measured at 115, 157 and 230 $\mathrm{MHz}$ using contiguous filters of width $10 \mathrm{kHz}$, and sweeping in frequency for 3, 5 and $9 \mathrm{MHz}$ respectively. The shapes are adequately described as truncated exponentials of width $\Delta t$ to $1 / e$. The rise time is essentially instantaneous - the apparent rise times of about one channel can be attributed to arbitrary positioning of the channels relative to the leading edge of the pulse. $\Delta t$ was measured by comparing relative intensities of adjacent channels on the trailing side of the pulse.

In Figure $2 \Delta t$ is plotted as a function of frequency. No correction has been made for the intrinsic pulse width which is $\sim 0.1 \mathrm{msec}$ at higher frequencies (Rankin et al., 1970). Between 115 and $230 \mathrm{MHz} \Delta t$ varies as $\lambda^{\beta}$ where $\beta=3.6 \pm 0.2$ (rms). The pulse shapes and wavelength dependence of the width are consistent with the truncated

* Operated by Associated Universities, Inc., under contract with the U.S. National Science Foundation. 
exponential shape and $\beta=4$ predicted by the theory of electron-scattering in the interstellar medium (Cronyn, 1970). As to whether the scattering occurs uniformly along the line of sight, or is dominated by one or two regions of inhomogeneity or by irregularities within the Crab Nebula itself can be determined by comparing $\Delta t$ with the observed angular diameter of NP 0531. Assuming that the small diameter source in the Crab Nebula is identical with NP 0531, present results indicate that the scattering is interstellar.
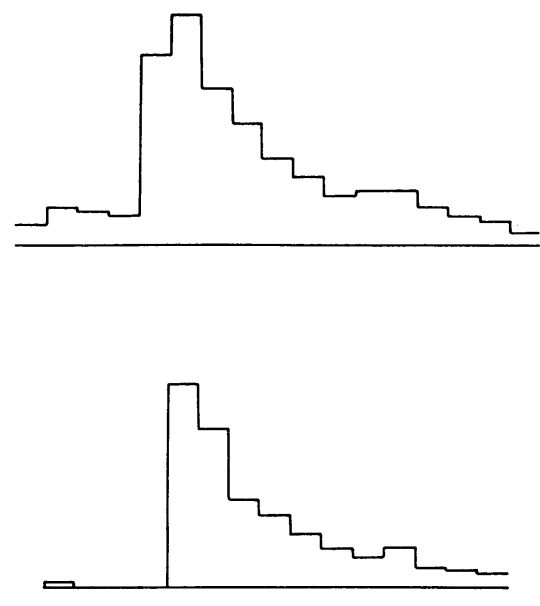

$115 \mathrm{MHz}$
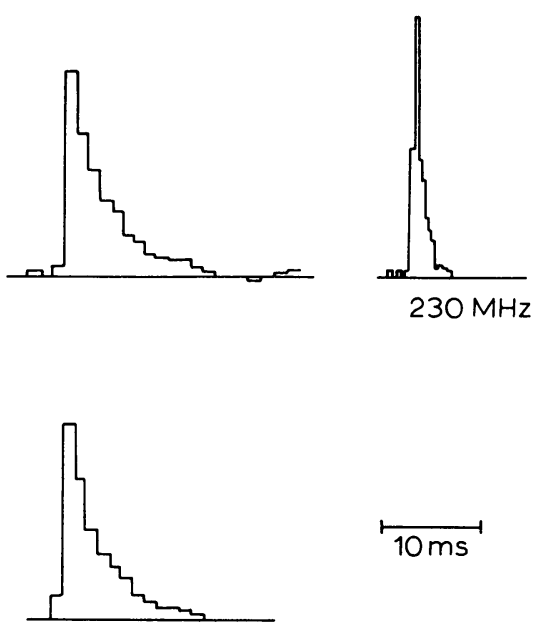

$157 \mathrm{MHz}$

Fig. 1. Shapes of different strong pulses from NP 0531 measured at different frequencies. All pulses are plotted with the same time scale.

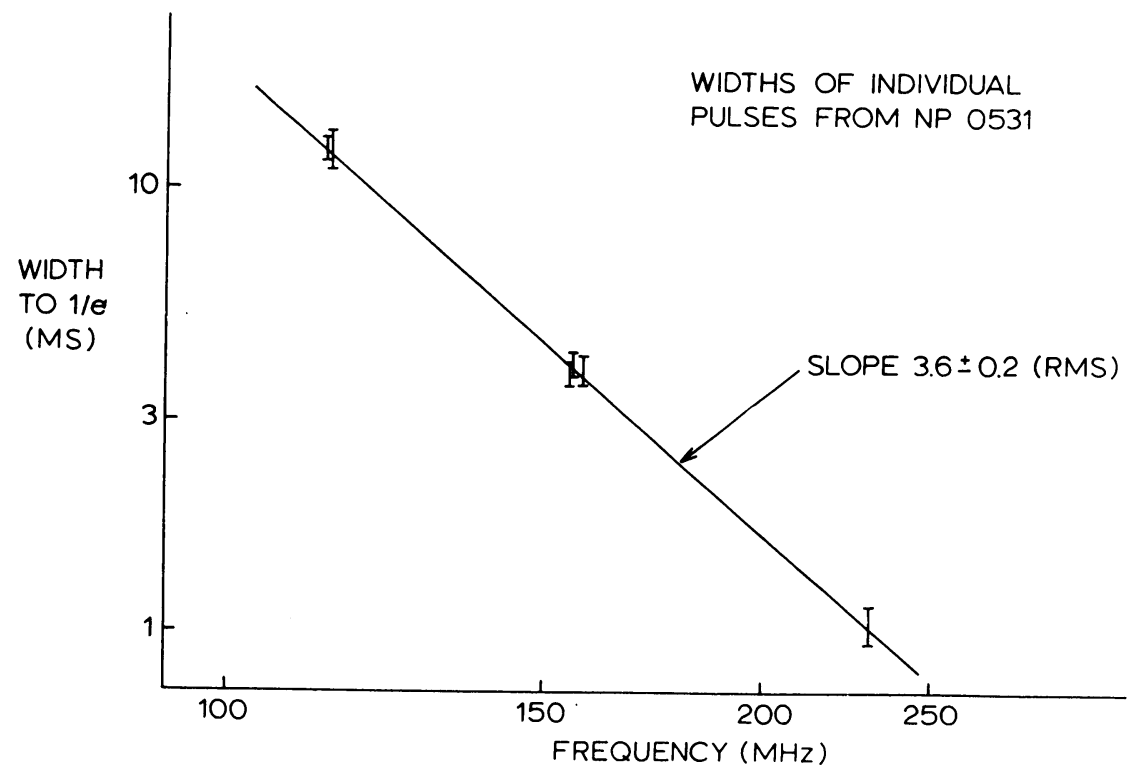

Fig. 2. Variation of 1/e decay time with frequency for strong pulses from NP 0531 . 
In a second experiment we used the swept frequency machine to record 59000 consecutive pulses from NP 0531 . Observing at $160 \mathrm{MHz}$ with filters of resolution $30 \mathrm{kHz}$, the time resolution was $3.46 \mathrm{msec}$ per channel, so that 5 pulses were observed simultaneously across the 50 channels of the filter bank. These 5 pulses were tracked for $0.56 \mathrm{MHz}$ and the sweep was recommenced every 3.500 pulsar periods. In this way $30 \%$ of the pulses were observed on two sweeps, while the remainder were observed on only one. The choice of 'pseudo-period' for the observations $(2 \times 3.500$ pulsar periods) ensured that in the subtraction of the comparison sweep from the pulsar sweep (see Sutton et al., 1970), corresponding parts of successive pulses were not subtracted from one another. However there is a partial subtraction of interpulses from main pulses, and vice versa.

Rankin et al. (1970) have shown that the average pulse shape of NP 0531 at 430 $\mathrm{MHz}$ contains three major components - the main pulse of width $300 \mu \mathrm{sec}$ which is followed $13.4 \mathrm{msec}$ later by the interpulse of width $400 \mu \mathrm{sec}$, and is preceded $1.6 \mathrm{msec}$ earlier by the $100 \%$ linearly polarized precursor of width $\sim 1 \mathrm{msec}$. At lower frequencies the average pulse profile is greatly smoothed by the interstellar scattering described above.

Figure 3 shows a histogram of observed intensity for the main pulse at $160 \mathrm{MHz}$ based on 27000 pulses of interference-free data. By restricting attention to 33 of the 50 filter bank channels, each pulse was counted only once. The intensities are integrations over a $3.46 \mathrm{msec}$ window whose centre varies with equal probability over a range of $\pm 1.73 \mathrm{msec}$ about the main pulse. Thus they are not true measures of intensity for individual pulses but are quite adequate for statistical purposes. Due to the pulse broadening there is some contamination from the precursor, and also from interpulses in the comparison sweep which will tend to produce negative intensities.

The combined contributions of the sky, the receiver and the Crab Nebula to the system temperature are about 90 units of intensity, of which the Crab Nebula contributes about 50 units.

Observations of strong radio sources showed that the recorded intensities were proportional to the power received. As described by Lovelace and Craft (1968), the observed histogram is then the convolution of the true distribution with some noise distribution. Also, because of the comparison sweep technique used, the noise distribution must be symmetric.

The histogram consists of two populations:

(1) A low intensity distribution which is symmetric about 1.2 units of intensity. The broadening can be attributed entirely to a Gaussian noise distribution of standard deviation 2.8 units, which is consistent with the system temperature. The pulse distribution is clearly narrow and includes many pulses with non-zero energy. Beyond this its shape is uncertain.

(2) A high intensity tail, which can conveniently be described as jumbo pulses. Assuming an exponential distribution extrapolated back to zero intensity, this contains $\sim 0.4 \%$ of the pulses and $\sim 5 \%$ of the pulsed energy.

This histogram is quite unlike those published for other pulsars which typically 
show a single population of exponential shape (Lovelace and Craft, 1968; Lang, 1969). However it should be pointed out that this histogram is produced by averaging pulse intensities over a frequency range considerably greater than the expected decorrelation frequency, thus smoothing out the effects of interstellar scattering. Most published histograms have been derived from observations with a narrow bandwidth in experiments purposely designed to measure the effects of interstellar scattering. For all pulsars there is clearly a need for measurements of intrinsic intensity histograms as

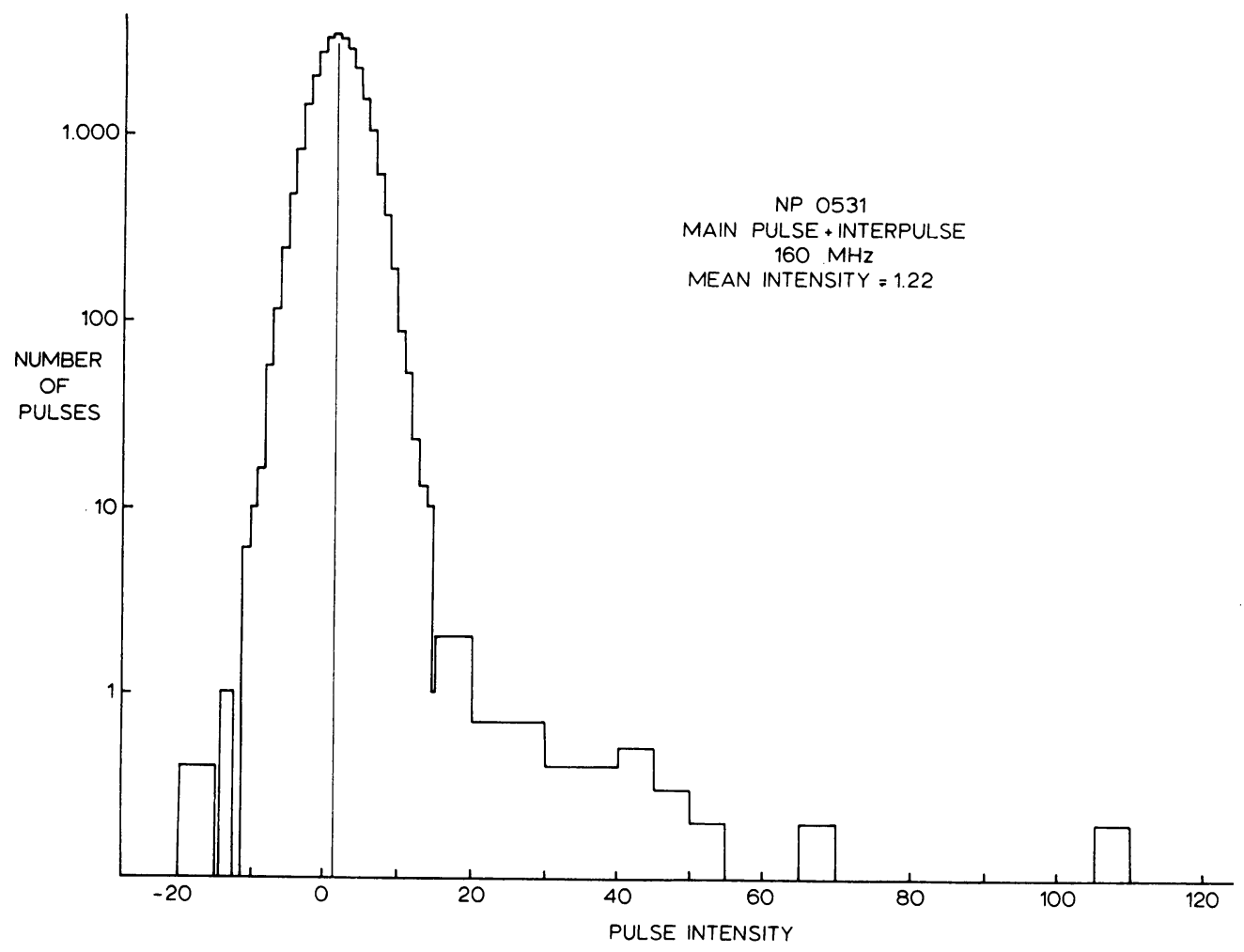

Fig. 3. Histogram of pulse intensity for 27000 main pulses of NP 0531 observed at $160 \mathrm{MHz}$. Intensities are integrals over a window of $3.46 \mathrm{msec}$ centred approximately on the main pulse, and are measured in arbitrary units. The mean intensity is 1.22 units. The cell size is 1 unit, but beyond 15 units the value plotted is the average over 5 cells. The vertical line indicates the axis of symmetry for the low intensity part of the distribution.

opposed to those produced by the interstellar medium. For example, Jones and Wielebinski (1969) overcame the difficulty of observing with a relatively narrow bandwidth by normalizing pulse intensities relative to the average of the adjoining pulses. Ten thousand pulses should be sufficient to establish the existence of a jumbo population similar to NP 0531.

Figure 4 shows arrival times of all pulses stronger than 16 units of intensity on the 
scale of Figure 3. All 59000 pulses were used. Although the time resolution was $3.46 \mathrm{msec}$, arrival times for the sharp leading edge could be estimated to the nearest $0.4 \mathrm{msec}$ using the relative intensities of the first two samples of the pulse, and assuming a truncated exponential pulse shape. There are clearly two groups of pulses, centred on the main pulse and the interpulse. No strong precursor pulses were observed although, within the limits of measurement, the possibility of one or two cannot be excluded. These results are a further example of the similarity of the main pulse and interpulse, and their differences from the precursor as regards pulse widths, optical radiation, $\mathrm{X}$-radiation and average linear polarization.

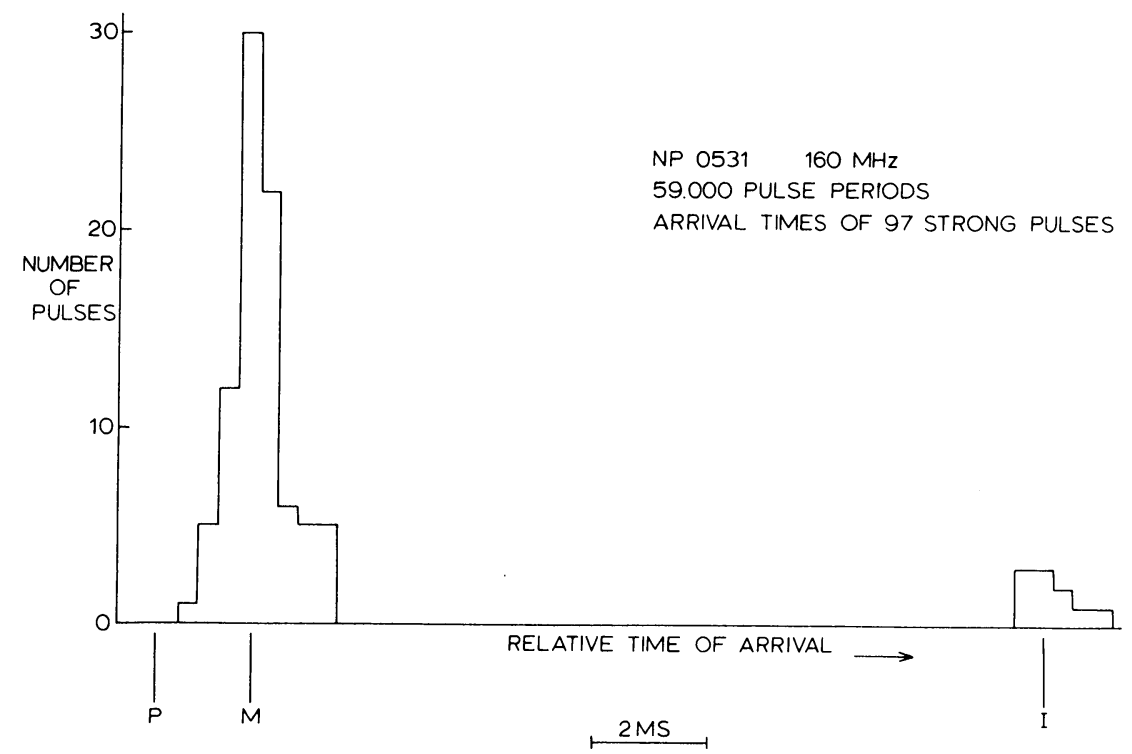

Fig. 4. Arrival times of 97 strong pulses, corresponding to all those stronger than 16 units of intensity (see Figure 3) but using all 59000 pulses. The relative arrival times of the precursor $(P)$, main pulse $(M)$ and interpulse $(I)$ are also shown.

The arrival times of these jumbo pulses were examined for periodicities, for bunching in time, and for consecutive main pulses and interpulses. For this purpose all intensities were corrected for the positioning of the window relative to the main pulse, and for small variations of antenna gain with hour angle. The resultant sample contained 50 pulses and was complete above a specified threshold. There is no evidence of any correlation in intensity between adjacent or nearby pulses or interpulses, or over longer intervals of time. It appears that the arrival times of jumbo pulses are independent of one another and are random in a Poissonian sense.

The pulse broadening due to scattering precludes measurements of intrinsic pulse widths or peak temperatures. However, the integrated energies of the strongest pulses can be compared with those at $430 \mathrm{MHz}$ (Heiles et al., 1970). In a sample of 174500 pulses Heiles et al. detected two pulses with energies $>2 \times 10^{-26} \mathrm{~J} \mathrm{~m}^{-2} \mathrm{~Hz}^{-1}$, and 
several with energies slightly less. In 59000 pulses at $160 \mathrm{MHz}$ the three largest have energies of 39,32 and $28 \times 10^{-26} \mathrm{~J} \mathrm{~m}^{-2} \mathrm{~Hz}^{-1}$. Rankin et all (1970) measured the spectral index of NP 0531 as $-2.6 \pm 0.3$. It follows that, within the errors of measurement, the ratios of peak pulse energies at the two frequencies are the same as the ratios of average pulse energies. Although there is evidence of wideband enhancement of NP 0531 pulses from 111 to $74 \mathrm{MHz}$ (Comella et al. 1968) and from 172 to $112 \mathrm{MHz}$ (Goldstein and Meisel, 1969), there is no evidence that the largest pulses at 430 $\mathrm{MHz}$ are also the largest at $160 \mathrm{MHz}$. Finally, assuming that the intrinsic pulse width at $160 \mathrm{MHz}$ is $\sim 0.1 \mathrm{msec}$, the same as that observed by Heiles et al. at 430 $\mathrm{MHz}$, the peak brightness temperature at $160 \mathrm{MHz}$ is $\sim 10^{33} \mathrm{~K}$.

The two populations of pulse intensity can be explained qualitatively by a beaming process in which the beam contains a strong narrow peak superimposed on a broad base and the orientation of this feature changes randomly from pulse to pulse in such a way that only occasionally is it directed at the Earth. This could arise from wideband maser amplification in which the direction of maximum amplification varies due to refraction in the amplifying plasma. In such a case it is unlikely that the largest pulses at 160 and $430 \mathrm{MHz}$ will be correlated.

The work of D. H. Staelin and R. M. Price is supported principally by grants from the U.S. National Science Foundation.

\section{References}

Comella, J. M., Craft, H. D., Lovelace, R. V. E., Sutton, J. M., and Tyler, G. L.: 1969, Nature 221, 453.

Cronyn, W. M.: 1970, Science 168, 1453.

Goldstein, S. J. and James, J. T.: 1969, Astrophys. J. 158, L179.

Heiles, C., Campbell, D. B., and Rankin, J. M.: 1970, Nature 226, 529.

Jones, B. and Wielebinski, R.: 1970, Astrophys. Letters 5, 17.

Lang, K. R.: 1969, Science 166, 1401.

Lovelace, R. V. E. and Craft, H. D.: 1968, Nature 220, 875.

Rankin, J. M., Comella, J. M., Craft , H. D., Richards, D. W., Campbell, D. B., and Counselman, C. C. : 1970, Astrophys. J. 162, 707.

Staelin, D. H., Reifenstein, E. C.: 1968, Science 162, 1481.

Staelin, D. H., Sutton, J. M.: 1970, Nature 226, 69.

Sutton, J. M., Staelin, D. H., Price, R. M. and Weimer, R.: 1970, Astrophys. J. 159, L89. 